\title{
Transfer of maternal immunity to piglets is involved in early protection against
} Mycoplasma hyosynoviae infection

Lauritsen, Klara Tølbøl; Hagedorn-Olsen, Tine; Jungersen, Gregers; Riber, Ulla; Stryhn, H.; Friis, N.F.; Lind, Peter; Kristensen, B.

Published in:

Veterinary Immunology and Immunopathology

Link to article, DOI:

10.1016/j.vetimm.2016.12.002

Publication date:

2017

Document Version

Peer reviewed version

Link back to DTU Orbit

Citation (APA):

Lauritsen, K. T., Hagedorn-Olsen, T., Jungersen, G., Riber, U., Stryhn, H., Friis, N. F., Lind, P., \& Kristensen, B. (2017). Transfer of maternal immunity to piglets is involved in early protection against Mycoplasma hyosynoviae infection. Veterinary Immunology and Immunopathology, 183, 22-30.

https://doi.org/10.1016/j.vetimm.2016.12.002

\section{General rights}

Copyright and moral rights for the publications made accessible in the public portal are retained by the authors and/or other copyright owners and it is a condition of accessing publications that users recognise and abide by the legal requirements associated with these rights.

- Users may download and print one copy of any publication from the public portal for the purpose of private study or research.

- You may not further distribute the material or use it for any profit-making activity or commercial gain

- You may freely distribute the URL identifying the publication in the public portal 
1 Transfer of maternal immunity to piglets is involved in early

2 protection against Mycoplasma hyosynoviae infection

5 K. Tølbøll Lauritsen ${ }^{1,2, *}$, T. Hagedorn-Olsen ${ }^{1}$, G. Jungersen ${ }^{1}$, U. Riber ${ }^{1}$, H. Stryhn $6{ }^{3}$, N. F. Friis ${ }^{1 \wedge}$, P. Lind ${ }^{1}$ and B. Kristensen ${ }^{2}$

9 Addresses of authors:

111 National Veterinary Institute, Technical University of Denmark, Bülowsvej 27, 1870 12 Frederiksberg C, Denmark

$13{ }^{2}$ Department of Disease Biology, Faculty of Health and Medical Sciences, University of 14 Copenhagen, Stigbøjlen 4, 1870 Frederiksberg C, Denmark

$15{ }^{3}$ Department of Health Management, University of PEI, Charlottetown, Prince Edward Island, 16 Canada. C1A 4P3 Canada

$18 *$ Corresponding author: Section for Diagnostic and Scientific Advice, National Veterinary

19 Institute, Technical University of Denmark, Bülowsvej 27, 1870 Frederiksberg C, Denmark

20 E-mail: ktla@ vet.dtu.dk, Tel.: +45 358863 72, FAX; +45 35886230

22 Tine Hagedorn-Olsen present address: Dako Denmark A/S, Produktionsvej 42, 2600 Glostrup,

23 Denmark 
Birte Kristensen present address: Klinik for Veterinær Dermatologi, Skovalleen 31, 2880

Bagsværd, Denmark

${ }^{\wedge}$ Deceased. N.F. Friis approved the manuscript before he passed away.

\section{Abstract}

31 Mycoplasma hyosynoviae causes arthritis in pigs older than 12 weeks. The role of

32 colostrum in protection of piglets against $M$. hyosynoviae infection is not clear. Our

33 objective was therefore to investigate whether transfer of maternal immunity to

34 piglets was involved in early protection against the infection. Experimental infections

35 were carried out in three groups of weaners receiving different levels of M. hyosynoviae-specific colostrum components; Group NC derived from Mycoplasma free sows and possessed no specific immunity to M. hyosynoviae. Group CAb pigs, siblings of the NC group, received colostrum with M. hyosynoviaespecific antibodies immediately after birth. Group CCE pigs were born and raised by

40 infected sows and presumably had the full set of colostrally transferred factors, 41 including specific antibodies. When $4 \frac{1}{2}$ weeks old, all pigs were inoculated 42 intranasally with $M$. hyosynoviae. The course of infection was measured through 43 clinical observations of lameness, cultivation of $M$. hyosynoviae from tonsils, blood 44 and synovial fluid and observation for gross pathological lesions in selected joints. 
45 Specific immune status in the pigs was evaluated through detection of antibodies by

46 immunoblotting and measurement of M.hyosynoviae-specific T-cell proliferation.

47 The latter analysis may possibly indicate that $M$. hyosynoviae infection induces a T-

48 cell response. The CCE piglets were significantly protected against development of

49 lameness and pathology, as well as infection with $M$. hyosynoviae in tonsils, blood 50 and joints, when compared to the two other groups. Raising the CCE pigs in an

51 infected environment until weaning, with carrier sows as mothers, apparently made

52 them resistant to $M$. hyosynoviae-arthritis when challenge-infected at $4 \frac{1}{2}$ weeks of 53 age. More pigs in group NC had M. hyosynoviae related pathological lesions than in 54 group $\mathrm{CAb}$, a difference that was significant for cubital joints when analysed on joint type level. This finding indicates a partially protective effect of passively transferred M. hyosynoviae-specific colostral antibodies upon development of M. hyosynoviae 57 related pathology. Thus, the level of passive immunity transferred from sow to piglet 58 seems to provide, at least partial, protection against development of arthritis. It cannot 59 be ruled out that the CCE pigs, by growing up in an infected environment, have had 60 the chance to establish an active anti-M. hyosynoviae immune response that 61 complements the maternally transferred immune factors. Evident from this study is 62 that the general absence of $M$. hyosynoviae arthritis in piglets can be ascribed mainly 63 to their immunological status. 


\section{Keywords}

66 Mycoplasma hyosynoviae, arthritis, colostrum, antibody, pig, lymphocyte

67 proliferation

70 Mycoplasma hyosynoviae infection is a common cause of acute and severe lameness

71 among Danish growing-finishing pigs (Nielsen et al., 2001). Herds with severely

72 affected pigs experience increased use of antibiotics and workload as well as reduced

73 animal welfare (Kobisch and Friis, 1996; Nielsen et al., 2001). The prevalence of $M$.

74 hyosynoviae in the Danish swine industry has not been investigated thoroughly,

75 however non-published experiences form Danish pig herds indicate that the majority 76 of these are infected.

78 M. hyosynoviae is harboured in the tonsils of infected pigs (Ross and Spear, 1973;

79 Friis et al., 1991). This carrier state is primarily established in pigs above ten weeks 80 of age and infection is rarely transmitted from sows to piglets (Hagedorn-Olsen et al., 81 1999a). Via the blood stream the mycoplasmas may spread to the joints (Kobisch and 82 Friis, 1996; Hagedorn-Olsen et al., 1999b) and cause arthritis in pigs above 12 weeks 83 of age (Ross and Duncan, 1970; Hagedorn-Olsen et al., 1999a). A previous 
experiment showed that 6-week-old pigs, immunologically naive with respect to $M$. hyosynoviae, were able to develop acute joint infection within 2 to 13 days after intranasal inoculation with the agent (Lauritsen et al., 2008). This indicated that the absence of $M$. hyosynoviae related lameness in this age group under field conditions must have another explanation than strictly age related factors.

Sow colostrum contains antibodies, which the newborn piglets absorb to the circulation through the gut (Frenyo et al., 1981; Klobasa et al., 1981; Rooke and Bland, 2002; Salmon et al., 2009; Bandrick et al., 2011; Nechvatalova et al., 2011), as well as other immunological components such as cells of the immune system, e.g. neutrophils and eosinophils, macrophages and lymphocytes (Evans et al., 1982; Schollenberger et al., 1986a; Schollenberger et al., 1986b; Magnusson et al., 1991, Nechvatalova et al., 2011). Live cells from sow colostrum are transferred across the gut epithelium of the piglet and into the blood/lymphatics (Tuboly et al., 1988; Williams, 1993; Salmon, 2000; Salmon et al., 2009; Nechvatalova et al., 2011) and it has been discussed in several papers whether colostral cells actively could comprise a pool of cellular immunocompetence that can be transferred from sow to the suckling piglet (Salmon, 2000; Wagstrom et al., 2000; Salmon et al, 2009; Nechvatalova et al., 2011). The role of colostrum in protection of piglets against $M$. hyosynoviae infection has so far not been clarified, although the abovementioned results by Lauritsen et al. (2008) may point in the direction of presence of maternally transferred immunity. 
105 Antibodies specific for $M$. hyosynoviae, presumably originating from colostrum, have 106 been shown to be present in suckling piglets (Blowey, 1993; Hagedorn-Olsen et al., 107 1999a). In the present study the hypothesis was that transfer of maternal immunity to 108 piglets is involved in early protection against Mycoplasma hyosynoviae infection. The 109 possible role of specific $M$. hyosynoviae antibodies was investigated by developing 110 an experimental colostrum model. One group of piglets were isolated from the sow 111 immediately after birth and fed cell-free colostrum containing significant levels of 112 specific $M$. hyosynoviae antibodies (Colostrum antibody group - CAb group). 113 Protection of this group after inoculation with M. hyosynoviae was compared to two 114 other groups, one that had suckled infected sows (Complete Colostrum and Exposure 115 group - CCE group) and one that had suckled sows that were immunologically naive 116 to M. hyosynoviae (Naive Colostrum group - NC group).

118 Materials and methods

\section{Animal material and housing conditions}

120 Thirty-two pigs were allocated to three groups, subjected to different regimens of

121 colostrum intake; $i$ ) the $\mathrm{CAb}$ group received $M$. hyosynoviae-specific antibodies via 122 colostrum that had been frozen and thawed to destroy live cells, ii) the NC group 123 suckled colostrum without $M$. hyosynoviae-specific immunity and was therefore 124 immunologically naive, iii) the CCE group received complete colostrum containing 
125 antibodies and cellular components from their M. hyosynoviae infected mothers, and 126 was exposed to infected environment until weaning. The experimental design is 127 illustrated in Fig. 1. All pigs included in the study were cross-breds (offspring from 128 Danish Landrace/Yorkshire sows and Duroc or Hampshire boars). The CAb and NC 129 groups were kept isolated under experimental conditions from birth, whereas the 130 CCE group was transferred to the experimental facilities one week before inoculation 131 (Fig. 1). All pigs of the study were weaned at 3-31/2 weeks of age (Fig. 1). The pigs 132 were kept loose in pens with concrete floors and abundant straw-bedding. Fresh water 133 was supplied ad libitum through water nipples, and the pigs were fed factory-made 134 pelleted standard swine feed without addition of any antimicrobials.

\section{Preparation of the colostrum pool}

137 The colostrum artificially fed to the CAb group (Fig. 1) was prepared from colostrum 138 of four sows from a M. hyosynoviae infected herd, but not the same herd which 139 supplied pigs for the CCE group. Within 24 hours after parturition 300-600 ml of 140 colostrum was collected from each sow using the following method; Sows were 141 prepared for colostrum collection by i.v. injection of $20 \mathrm{IU}$ oxytocin $\left(\right.$ Oxytocin $^{\circledR}$, Leo 142 Vet) and the udder was washed and disinfected ( $0.5 \%$ chlorhexidin in $70 \%$ ethanol).

143 Colostrum was collected by hand stripping into sterile wide mouth glass bottles. After 144 removing 2-4 $\mathrm{ml}$ for later cultivation for M. hyosynoviae, $200 \mathrm{mg}$ tiamulin (Tiamutin 
$145{ }^{\mathrm{R}}$, Novartis) was added per $100 \mathrm{ml}$ colostrum and the colostrum was stored at $-20^{\circ} \mathrm{C}$.

146 Further, the colostrum was thawed, pooled, filtered through sterile gauze, aliquoted 147 into sterile bottles and stored at $-20^{\circ} \mathrm{C}$ until use. Before being fed to the newborn 148 pigs, the colostrum was thawed in a lukewarm water bath, and the temperature 149 adjusted to $38^{\circ} \mathrm{C}$. Further details on the colostrum feeding to piglets are described in 150 Fig. 1. All colostra used were cultivation negative for Mycoplasma spp prior to 151 addition of Tiamulin.

\section{Inoculation with $M$. hyosynoviae}

154 Between 4 and $4 \frac{1}{2}$ weeks of age all pigs were inoculated with a cloned field strain of 155 M. hyosynoviae, Mp927 (titres $10^{7}$ to $10^{8}$ colour changing units (CCU) per ml). The 156 method used for preparation of inoculum is described by Lauritsen et al. (2008). Pigs 157 were inoculated intranasally into the dorsal meatus, while in dorsal recumbency. 158 Inoculation dose was $1 \mathrm{ml}$ in each nostril.

160 Mycoplasma cultivation

161 Cultivation for M. hyosynoviae from heparin-stabilized blood samples was performed 162 on post inoculation day (PID) 4, 7, 9, 12 and 15. Tonsillar scrapings, obtained with a 163 sterile blunt steel scraper especially designed for the purpose, were collected two 164 days before inoculation and on PID 4, 7, 9 and 12. Cultivation for M. hyosynoviae in 
165 colostrum was performed in 1:10 serial dilutions to $10^{-4}$ in modified Hayflick's 166 medium (Kobisch and Friis, 1996). All other methods, used in this study for 167 mycoplasma cultivation, including production of inoculation material, have been 168 described by Lauritsen et al. (2008).

\section{Clinical recordings and post mortem examinations}

171 Prior to inoculation all pigs had a normal body condition and did not show any 172 clinical signs of disease. Every day post inoculation, the pigs were observed for 173 clinical signs of lameness and other signs of disease. The pigs were euthanized and 174 autopsied on PID 12, 14 or 16, i.e. in the time period of expected occurrence of the 175 acute infection phase (Kobisch and Friis, 1996; Hagedorn-Olsen et al., 1999c). The 176 date of euthanasia for each pig was determined before inoculation and pigs from each 177 group were evenly represented on the necropsy days. Euthanasia was performed by 178 stunning with a captive bolt pistol followed by exsanguination. At autopsy, six joints 179 per pig were examined for gross pathological lesions since we focused on cubital, 180 stifle and tibiotarsal joints. For each joint the conditions of the synovial fluid and 181 synovial membrane were evaluated by scoring the following seven variables: 182 Synovial fluid colour, volume and transparency, Synovial membrane edema, 183 hyperaemia, hypertrophy and discolouration. In addition the joint cartilages were 184 examined for lesions and discolouration. A pathoanatomical diagnosis was made for 
185

186

187

188

189

each joint based on the sum of all macroscopic findings recorded for the joint. Synovial fluid was collected aseptically for $M$. hyosynoviae cultivation from these joints and transferred to a sterile tube containing mycoplasma transport medium (Kobisch and Friis, 1996). The amount of synovial fluid used for cultivation varied depending on the amount obtainable from the joints - from one drop to one ml. The synovial fluid samples were also examined for the presence of $M$. hyorhinis, M. hyopneumoniae and M. flocculare by cultivation. From each pig, the tonsils were collected for M. hyosynoviae cultivation.

The procedures related to animal experimentation had been approved by the Danish Animal Experiments Inspectorate (Licence No. 1999/561-207).

\section{M. hyosynoviae antigen for lymphocyte proliferation assay and immunoblots}

Pelleted (1.6 g) M. hyosynoviae species type strain S16 (Ross and Karmon, 1970) resuspended in $10 \mathrm{ml}$ sterile Milli Q water was subject to 10 repeated freeze-thaw cycles and finally centrifugated at $1000 \mathrm{x} \mathrm{g}, 30 \mathrm{~min}$. The washed pellet was solubilized twice on ice in NP40 lysis buffer (2\% v/v Nonidet P40, 2 mM EDTA, 0.1 mM IAA and $1 \mathrm{mM}$ PMSF in PBS), and centrifuged at $20000 \mathrm{x}$ after which the new pellet was boiled for 5 minutes in $3 \mathrm{ml}$ lysis buffer with addition of $2 \% \mathrm{w} / \mathrm{v}$ SDS and centrifuged at $20000 \mathrm{x}$ g for $30 \mathrm{~min}$. Free SDS was removed by ultrafiltration through an YM 10 (Amicon) membrane at $4^{\circ} \mathrm{C}$. The resulting antigen solution was called 
205 Mhyos-antigen. It was aliquoted and stored at $-20^{\circ} \mathrm{C}$. A protein concentration of 3.3 $206 \mathrm{mg} / \mathrm{ml}$ was measured by the Micro BCA (bicinchoninic acid) Protein Assay (Pierce) 207 using bovine serum albumin as standard. To assure that the antigen exerted no 208 inhibitory effect on cell cultures, an MTT test (Mosmann, 1983) for antigen toxicity 209 and non-specific stimulation was performed. The antigen preparation induced low 210 non-specific activity at $20 \mu \mathrm{g} / \mathrm{ml}$. However, at lower concentrations no non-specific 211 activity was observed and the antigen was non-toxic at all concentrations.

\section{BrdU lymphocyte proliferation assay}

214 Antigen-specific lymphocyte proliferation in response to $M$. hyosynoviae challenge 215 infection was investigated in blood samples from all pigs two days before inoculation 216 and on PID 7 and 12. Proliferation was measured by flow cytometry, assessing cells 217 that had incorporated the thymidine analog Bromo-deoxy-Uridine (BrdU) in newly 218 synthesized DNA (Riber and Jungersen, 2007). Briefly, peripheral blood 219 mononuclear cells (PBMCs, $3 \times 10^{6} / \mathrm{ml}$ ) in cell culture medium (RPMI 1640 with 220 GlutaMAX ${ }^{\mathrm{TM}} \mathrm{I}$, foetal calf serum (10\%), penicillin $(100 \mathrm{U} / \mathrm{ml})$, streptomycin (100 $221 \mu \mathrm{g} / \mathrm{ml}$ )) were incubated in 24 well, cell culture plates (Greiner Labortechnik GmbH, 222 Germany): SEB-culture (Staphylococcal enterotoxin B, $5 \mu \mathrm{g} / \mathrm{ml}$, Alexis, Grünberg, 223 Germany), Ag-culture (Mhyos-antigen, $10 \mu \mathrm{g} / \mathrm{ml}$ ), RPMI-culture (nil-stimulation). 224 Incubation was performed for 5 days at $37^{\circ} \mathrm{C}$ in $5 \% \mathrm{CO}_{2}$, the last 18 hours with 
225 addition of 5-Brom-2'-Deoxyuridine (BrdU $60 \mu \mathrm{M}$, Sigma-Aldrich, St. Louis, MO, 226 USA).

227 Cells were harvested and stained with mAb against swine CD3 (clone PPT3, Yang et 228 al 1996) and secondary R-phycoerythrin conjugated antibody (R0439, DAKO, 229 Denmark). Then cells were fixed with BD-lysis-solution (BD biosciences) and 230 permeabilized with BD-permeabilizing-solution (BD Biosciences) and stained with 231 FITC conjugated Mab against BrdU containing DNAse (BD Biosciences). As control 232 for BrdU staining, cells were incubated with isotype-control antibody (X0927, 233 DAKO, Denmark). Cells were analysed on FACScan by use of CellQuest software 234 (BD Biosciences).

23520000 gated cells (interpreted as live lymphocytes) were acquired and CD3+BrdU+ 236 double positive cells, i.e. T-cells that have proliferated, were measured (see 237 supplementary material for details). Mhyos-antigen-specific lymphocyte proliferation 238 was calculated as: $\% \mathrm{CD} 3+\mathrm{BrdU}+$ cells (Ag-culture) with subtraction of $239 \% \mathrm{CD} 3+\mathrm{BrdU}+(\mathrm{RPMI}-\mathrm{culture})$.

\section{Detection of antibodies}

241 Sera from the pigs collected before inoculation (when pigs were 2-3 days, 2 and 4 242 weeks of age) and colostrum samples from sows no. 1-4 were tested by 243 immunoblotting for the presence of specific antibodies against M. hyosynoviae; The 244 Mhyos-antigen was diluted in sample buffer (4× NOVEX NuPAGE Sample Buffer, 
245 San Diego, CA) containing 100mM $\beta$-mercaptoethanol. For the electrophoresis, 246 NuPAGE 4-12\% Bis-Tris gels (NOVEX) were used in running buffer (20× NOVEX 247 NuPAGE MOPS SDS Running Buffer). SeeBlue PreStained Standards (NOVEX) 248 were used as marker. Blotting was performed in NuPAGE Transfer Buffer (NP0006, 249 NOVEX) and membranes were blocked with TBS + $0.5 \%$ Tween-20. Nitrocellulose 250 strips cut from the blots were incubated overnight with either serum or colostrum 251 (dilutions 1:200 or 1:500 in TBS + 0.5\% Tween-20, respectively). HRP-conjugated 252 rabbit anti-swine Ig antiserum (DAKO cat.no. P164, 1:2000 in TBS + 0.5\% Tween253 20) was used as secondary antibody. Between each step, the strips were washed with 254 TBS $+0.5 \%$ Tween-20. Finally the strips were washed for 10 minutes in $50 \mathrm{mM}$ 255 sodiumacetate and the protein bands were developed in dioctyl sodium sulfasuccinate 256 (DSS)/tetramethylbenzidine-solution for 1 to 15 minutes. The strips were then 257 washed in a DSS-solution for maximum 15 minutes and finally dried, after which the 258 presence of $M$. hyosynoviae-specific bands was evaluated. Defining the bands that 259 were specific for M. hyosynoviae was performed by comparing Western blot band 260 patterns obtained with serum of experimentally infected pigs (sera supplied by Dr. 261 Niels Filskov Friis). Recognition of two bands at level with the 191kDa size marker 262 (Fig. 2) was consistent in all expectedly positive pigs and was absent in naive pigs 263 (data not shown) as well as in pigs infected with other swine specific mycoplasmas. 264 These two bands were used for differentiating between seropositive and seronegative 265 pigs (Fig. 2). 
267 Additionally the total Ig content in serum from 2 to 5 days old pigs was measured in a 268 non-competitive direct ELISA, using plates coated with rabbit anti-swine Ig 269 antiserum (DAKO Z0139). As secondary antibody HRP-conjugated, rabbit anti270 swine Ig antiserum (DAKO P0164) was used. Ig concentration in serum samples was 271 calculated from a standard curve of two-fold dilutions of normal swine Ig fraction 20 $272 \mathrm{mg} / \mathrm{ml}$ (DAKO X0906) (start dilution $3.125 \mathrm{ng} / \mathrm{ml})$.

\section{Statistical analysis}

275 Multiple measurements on the same pig were, whenever possible without substantial 276 loss of information, aggregated into a single measure reflecting the overall status of 277 the pig. This approach facilitates the biological interpretation of the results and avoids 278 complex modelling of discrete repeated measures outcomes (Diggle et al., 2002). 279 Cultivation of blood samples and recordings of clinical signs of lameness during the 280 period from challenge to autopsy were interpreted in parallel, that is, the pig was 281 considered a positive reactor if at least one recording was positive. Cultivation of 282 tonsillar samples at autopsy were positive for almost all pigs, and an additional 283 analysis was therefore carried out for records of whether pigs had only positive 284 samples (negative interpretation in parallel). Autopsy results (synovial fluid 285 cultivations, pathological findings) for multiple joints were both interpreted in 
parallel across joints and analysed separately for cubital, stifle and tibiotarsal joints because $M$. hyosynoviae arthritis seems to be more frequently observed in some joints than in others (Ross, 1973). As the majority of joints in two of the groups showed no pathological lesions, a presence/absence recording of lesions was preferred over using scores of the individual arthritis severity.

The statistical procedure used to compare the groups with respect to dichotomous outcomes at the pig level was a logistic regression controlling for confounding of experiment ( 1 or 2), litter and day of measurement (autopsy recordings only) by fixed effects. The potential confounders were omitted when statistically clearly nonsignificant ( $>0.10)$ and without any substantial confounding effect (less than $20 \%$ 297 change in odds-ratio (Dohoo et al., 2009)). The odds-ratio expresses roughly the 298 factor by which the occurrence of $M$. hyosynoviae related findings was higher in one 299 group (e.g., NC) relative to another group (e.g., CAb). The effectiveness of 300 controlling for experiment was confirmed by additional Mantel-Haenszel analyses 301 and Generalised Estimating Equation (GEE) logistic regression (Davis, 2002) with an 302 exchangeable correlation structure. Analyses for the NC and CAb groups of synovial 303 fluid and arthritis outcomes at multiple joints used a similar GEE logistic regression 304 to account for two joints (of each type) being measured in each pig. In addition to the 305 logistic regression analyses, Fisher's exact test was used for outcomes that were 306 constant within at least one group. As described by Greenland et al. (2016), we 
307 interpreted the p-values as continuous measures that express the compatibility 308 between the data and the statistical model used. The significance level was set at $309 \mathrm{p}<0.05$. Some findings close to statistical significance were noted as such because of 310 their potential interest, but they were not treated as significant results in the 311 discussion and conclusion. All analyses were carried out by the statistical software 312 SAS, version 9.

314 T-cell proliferation was compared among the three groups by the non-parametric 315 Kruskal-Wallis test, supplemented with comparisons between selected pairs of 316 groups by the non-parametric Mann-Whitney test, using GraphPad Prism version 317 5.02, GraphPad Software, San Diego California USA, www.graphpad.com.

319 Results

\section{Clinical signs}

321 Among the pigs in the $\mathrm{NC}$ and $\mathrm{CAb}$ groups there were some registrations of lameness

322 post challenge (Table 1). The CCE group had no lameness registrations and was 323 found to differ significantly from the NC group ( $\mathrm{p}=0.007)$ (Table 1). The difference 324 between group $\mathrm{NC}$ and group $\mathrm{CAb}$ was not statistically significant $(\mathrm{p}=0.22)$. 


\section{Gross pathological findings}

327 The observed gross pathological lesions were of varying severity ranging from slight

328 serous arthritis (slightly increased volume of synovial fluid that might be discoloured

329 and turbid. In synovial membrane: mild edema, hyperaemia and/or discolouration),

330 hyperplastic arthritis (varying degree of increase in both synovial fluid volume, 331 discolouration and turbidity. The synovial membrane showed pronounced 332 hyperplasia, sometimes edema, and quite often hyperaemia and discolouration) or 333 serofibrinous arthritis (significantly increased synovial fluid volume with some 334 discolouration and pronounced turbidity, the synovial membrane had marked edema, 335 and some discolouration). Several pigs in groups NC and CAb had M. hyosynoviae 336 related pathological lesions, a marked difference to group CCE with no such findings 337 (Table 1). For all joint types, more pigs in group NC had pathological lesions than in 338 group $\mathrm{CAb}$. The difference was statistically significant for cubital joints (odds339 ratio $=29, \mathrm{p}=0.002$ ) and close to significant for stifle joints (odds-ratio $=6.4, \mathrm{p}=0.095$ )

340 (Table 2). For tibiotarsal joints, the day of autopsy had a significant effect; day 16 341 post challenge had a higher occurrence of pathological lesions than preceding days 342 (odds-ratio $=14, \mathrm{p}=0.003$ ). 


\section{Synovial fluid cultivations}

345 Cultures of synovial fluid from the joints from all pigs in group CCE were negative

346 for M. hyosynoviae while M. hyosynoviae was demonstrated in joints from the 347 majority of pigs in both groups NC and CAb (Table 1). For both cubital, stifle and 348 tibiotarsal joints, more pigs had positive cultures in group $\mathrm{NC}$ than in group CAb. 349 These differences were not statistically significant (Table 2), even if the comparison 350 between $\mathrm{NC}$ and $\mathrm{CAb}$ groups for stifle joints was close to significant (odds-ratio 351 >1000, p=0.086). M. hyorhinis, M. hyopneumoniae and M. flocculare were not 352 isolated from any of the synovial fluid samples.

\section{Cultivation of M. hyosynoviae from tonsils and blood}

355 Prior to inoculation tonsillar scrapings from all pigs were $M$. hyosynoviae negative 356 except from one pig in group CCE. Contrary to this, all but one pig from the CCE 357 group, were tonsil carriers at autopsy (Table 1). While almost all pigs in groups NC 358 and CAb were positive on all repeated samplings post inoculation, the pigs in group 359 CCE had any number between 0 and 5 (maximum) positive samplings. One 360 interpretation of this pattern is that pigs in the CCE group tended to develop a carrier 361 state later than pigs of the other groups. This was reflected in a significant difference 362 between the CCE group and the two other groups but no significant difference 
363 between the latter groups, when analysing positive cultivations on PID 4 (results not 364 shown).

366 Group CCE pigs had no positive blood samples during the experiment while all pigs 367 in groups $\mathrm{NC}$ and $\mathrm{CAb}$ experienced a haematogenous phase (Table 1). The majority 368 of pigs in the NC and CAb groups had the same pattern of cultivation positive blood 369 samples, with the first three samples (PID 4, 7 and 9) being culture positive.

\section{Lymphocyte proliferation}

The T-cell proliferation assay was implemented in a previous experiment including four M. hyosynoviae inoculated pigs (13 weeks old) and one non-inoculated control

374 pig (unpublished data, pigs described by Lauritsen et al. (2008)). Signs of specific 375 lymphocyte proliferation against Mhyos-antigen were found on PID 11 in the four 376 inoculated pigs $\left(\mathrm{CD}^{+}{ }^{+} \mathrm{BrdU}^{+}\right.$cells: $\left.1.4 \% ; 2.2 \% ; 7.2 \% ; 7.6 \%\right)$, but not in the control 377 pig $(0.81 \%)$. No differences in level of proliferation were observed between Ag378 cultures with either $2 \mu \mathrm{g} / \mathrm{ml}$ or $10 \mu \mathrm{g} / \mathrm{ml}$ of Mhyos-antigen.

379 In the present study $\% \mathrm{CD}^{+} \mathrm{BrdU}^{+}$in RPMI-cultures varied quite a lot, and 380 particularly on PID 7 high $\% \mathrm{CD}^{+} \mathrm{BrdU}^{+}$was measured in RPMI-cultures in some 381 pigs from all groups (Mean: 4.6\%, Range 1.0-15.6\%), which in some cases could be 382 related to positive cultivation of $M$. hyosynoviae in blood samples. Contrary to this, 
383

most samples from PID 12 had a $\% \mathrm{CD}^{+}{ }^{+} \mathrm{BrdU}^{+}$in RPMI-cultures around 1\% (Mean: 1.4\%, Range $0.4-3.9 \%$ ), and only one pig had a M. hyosynoviae cultivation-positive blood sample on this day. Therefore, comparison of antigen-specific proliferative response in the three treatment groups was only performed at PID 12. As shown in Fig. 3, there was a large variation in degree of proliferation on pig level seen as an individual variation within the groups, concerning the antigen-specific proliferative response that we have measured on PID 12. We found no significant differences between the three treatment groups $(\mathrm{P}=0.35$, Kruskal-Wallis test $)$. The medians of the $\mathrm{CAb}$ and CCE groups (i.e. the groups appearing most different in Fig. 3) were not statistically different ( $\mathrm{P}=0.16$, Mann-Whitney test). Likewise, the difference in the medians of the $\mathrm{NC}$ and $\mathrm{CCE}$ groups was statistically non-significant $(\mathrm{P}=0.37)$ on $\mathrm{PID}$ 12.

\section{Antibody responses}

Evaluating the serum antibody profiles of pigs in the three groups by immunoblotting revealed that the NC group possessed no bands specific for M. hyosynoviae at any time prior to inoculation (Fig. 2). Contrary to this, immunoblots from all pigs of groups $\mathrm{CAb}$ and $\mathrm{CCE}$ revealed bands specific for $M$. hyosynoviae prior to inoculation, but the general band patterns of these two groups differed from one to another (Fig. 2). In accordance with the findings in the pigs, colostrum from the 
403

404

405

406

407

408

409

410

411

412

413

414

415

416

417

418

419

420

421

422

423

M. hyosynoviae immunologically naive sows (no. 1 and 2) had no M. hyosynoviae specific bands whereas specific bands were found in colostrum of sows no. 3 and 4 from the infected herds and in the colostrum pool used for feeding the piglets. The average total immunoglobulin content in serum from 2- to 5-day-old pigs, measured in ELISA, were for the CAb group $16 \mathrm{mg} / \mathrm{ml}$, the $\mathrm{NC}$ group $30 \mathrm{mg} / \mathrm{ml}$ and the CCE group $32 \mathrm{mg} / \mathrm{ml}$.

\section{Discussion and conclusion}

The result of the immunoblottings confirmed that the specific immune statuses of the pigs were the following on the day of inoculation with $M$. hyosynoviae; i) The pigs from sows that were immunologically naive (group NC) possessed no specific immunity against the agent. ii) The colostrum treated pigs (group CAb pigs) possessed specific antibodies as a consequence of the artificial colostrum administration. iii) The pigs from infected sows (group CCE) had received specific antibodies, and presumably the full set of maternally transferred factors from colostrum of their infected dams.

The course of infection after inoculation in the CCE group differed significantly from that of the two other groups for several parameters measured; no signs of clinical arthritis or gross pathological findings was found in the CCE group, and cultivation of M. hyosynoviae from tonsils, blood and joints of these pigs was significantly 
424 reduced compared to the other groups. With respect to immunological status and 425 M. hyosynoviae-infection status, the CCE group represent the population of newly 426 weaned pigs in most Danish herds. They possess $M$. hyosynoviae-specific antibodies 427 just as it has been shown to be the case for piglets from infected sows in Danish herds 428 (Hagedorn-Olsen et al., 1999a). Also the limited effect of the experimental infection 429 in the CCE pigs is in accordance with the observation that clinical $M$. hyosynoviae 430 arthritis does not affect pigs below 30-40 kg in infected herds (Kobisch and Friis, 431 1996). Raising the CCE pigs in an infected environment until weaning, with carrier 432 sows as mothers, apparently made these pigs resistant to $M$. hyosynoviae-arthritis, 433 when challenge infected at 4 weeks of age. However observational studies performed 434 in infected herds indicate that this, probably maternally-derived, protection is of 435 limited duration and that pigs become susceptible to $M$. hyosynoviae infection and are 436 at risk of developing M. hyosynoviae-related arthritis later in life (Ross and Spear, 437 1973; Hagedorn-Olsen et al., 1999a).

439 Previous intranasal inoculation experiments with $M$. hyosynoviae, performed in $M$. 440 hyosynoviae-free pigs, have shown that 13 to 17 -week-olds experienced a 441 generalisation phase from PID 2, with no detectable mycoplasmas in blood after PID 4429 (Hagedorn-Olsen et al., 1999b) and that 6-week-old pigs, immunologically naive 443 with respect to M. hyosynoviae, primarily had bacteremia at PID 4, 6 and 8 (Lauritsen 444 et al., 2008). This is in accordance with the findings in groups $\mathrm{NC}$ and $\mathrm{CAb}$, where 
445 positive blood cultivations were predominantly seen on PID 4, 7 and 9. The lack of 446 positive blood cultivations in group CCE pigs during the experiment, i.e. no 447 detectable generalisation from PID 4 and forth, indicates a very short period of, or 448 maybe a total lack of haematogenous spread of $M$. hyosynoviae in this group.

450 The experimental infection resulted in occurrence of clinical M. hyosynoviae-arthritis 451 both in pigs of group $\mathrm{NC}$ and group $\mathrm{CAb}$. Also gross pathological findings 452 compatible with $M$. hyosynoviae-infection, bacteremia and early tonsillar 453 colonization were observed in these groups. The presence of an ongoing disease 454 condition in the pigs of the NC group was also reflected by a drop in the negative 455 acute phase protein, transthyretin, 7 days after infection (Heegaard et al. 2011). The 456 higher frequency of $M$. hyosynoviae related pathological lesions in cubital joints upon 457 challenge infection in group $\mathrm{NC}$, as compared to group $\mathrm{CAb}$, may indicate a partially 458 protective effect of the intake of $M$. hyosynoviae-specific colostral antibodies upon 459 development of $M$. hyosynoviae related pathology. The average total immunoglobulin 460 content in serum from 2- to 5-day-old pigs, as measured in ELISA, showed that the 461 colostrum treated pigs possessed the lowest concentration of total $\operatorname{IgG}$ in serum. 462 Therefore the observed, partially protective, effect of the colostrum treatment on 463 development of arthritis can be ascribed to M. hyosynoviae specific antibodies, and 464 not to a generally higher level of non-specific maternally transferred 465 immunoglobulins. 
467 The role of specific antibodies in M. hyosynoviae infection is not clear, and could 468 include elimination of the mycoplasmas (neutralisation, opsonisation, complement 469 activation) as well as creation of pathology during infection (e.g. deposition of 470 immune complexes). The partially protective effect of $M$. hyosynoviae specific 471 antibodies on development of arthritis found in this study is contradictory to the 472 findings in pigs above 10 weeks made by Blowey (1993). He found that the 473 appearance of arthritis in gilts was independent of the level of specific antibodies in 474 serum, indicating that the antibody level was of lesser importance for protection 475 against the disease. Also conflicting herd observations, describing antibody levels in 476 relation to bacteremia with $M$. hyosynoviae, have been reported; Hagedorn-Olsen et 477 al. (1999a) found cases of pigs that had raised a specific serological response against 478 M. hyosynoviae, after which they developed a generalisation phase with the agent. 479 Contrary to this, Nielsen et al. reported that 3 to 5 -month-old pigs with bacteremia 480 had a lower level of $M$. hyosynoviae specific antibodies, than pigs with no 481 demonstrable bacteremia (Nielsen et al., 2005). The general ability of mycoplasmas 482 to vary their surface antigens to evade the host immune response (Razin et al., 1998) 483 may make antibody-mediated elimination difficult to obtain and could be an 484 explanation for the above mentioned ambiguous effect of antibodies. Thus the 485 importance of antibodies in protection against $M$. hyosynoviae arthritis is equivocal. 
487 It has not previously been investigated whether $M$. hyosynoviae infection induces a 488 specific T-cell response. In this study we found a marked variation between 489 individual pigs concerning specific T-cell responses post inoculation. However, T490 cell responses are in general widely fluctuating over time and between individuals, so 491 we would not expect all pigs to mount a synchronised and similar specific T-cell 492 response post inoculation. The high percentage of specific proliferation in response to M. hyosynoviae antigen found in some pigs on PID 12 indicates that M. hyosynoviae 494 infection has the potential of inducing an antigen-specific T-cell response. The 495 involvement of this response in protection against the infection remains uncertain, 496 however, it might be that a cell-mediated immune response participate in protection. 497 We found in this study no statistically significant difference between the three 498 treatment groups, with respect to percentages of $M$. hyosynoviae antigen-specific T499 cell proliferation.

501 The design of the study did not take differences in $M$. hyosynoviae strains into 502 account. Ross et al. (1978) demonstrated different $M$. hyosynoviae strains by 503 serological and electrophoretic methods, and Kokotovic et al. (2002a; 2002b) found a 504 pronounced genetic diversity in chromosomal fingerprints performed on Danish herd 505 isolates of $M$. hyosynoviae. In our study several strains could be involved; 1) the 506 inoculation material, 2) the strain(s) present in the herd that supplied the colostrum 
508 differences in band pattern between immunoblots of pigs in the CAb and the CCE 509 groups. However, the immunoblots reveal many bands of which we do not know how 510 many are M. hyosynoviae-specific. Thus we do not know whether we worked with at 511 homo- or heterologous system, or a mixture of the both, in this challenge experiment. 512 Designing the study in a way so that all infected sows were infected with a strain 513 similar to that of the inoculation material was neither economically nor practically 514 feasible.

516 Based on the results of our study, we conclude that in contrast to immunologically 517 naive piglets, piglets that have been raised in an infected environment and have 518 suckled infected sows are protected against early infection with $M$. hyosynoviae and 519 development of arthritis when challenge infected at $4 \frac{1}{2} 2$ weeks of age. We found 520 indications that this protection is related to the level of passive immunity because $M$. 521 hyosynoviae-specific maternally transferred antibodies provided at least partial 522 protection against development of arthritis in otherwise immunologically naive pigs. 523 For comparison, a similar protective effect of colostral antibodies against another 524 mycoplasma, Mycoplasma hyopneumoniae, has been described by Rautiainen \& 525 Wallgren (2001), Wallgren et al. (1998) and Siblia et al. (2008). However the marked 526 difference between groups $\mathrm{CAb}$ and $\mathrm{CCE}$ indicates that something more than 527 maternally derived antibodies contributes to the protection. It cannot be ruled out that 528 the CCE pigs, by growing up in an infected environment, have had the chance to 
529 establish an active immune response against the agent that complements the 530 maternally transferred immune factors, although only one of the 13 pigs in this group 531 was cultivation positive in the tonsils prior to inoculation. Alternatively the observed 532 high level of resistance to the challenge infection in the pigs that had suckled infected 533 sows could be due to the uptake of cellular components, e.g. primed lymphocytes, 534 from the fresh sow colostrum. By using a model antigen, Nechvatalova et al. (2011), 535 showed that sows via colostrum were able to transfer antigen-specific lymphocytes to 536 the mesenteric lymph nodes and blood stream of their offspring. Likewise Hlavova et 537 al. (2014), found that colostrum contained high numbers of antigen-experienced 538 lymphocytes with a central/effector memory function, that might play a role as 539 passive immunity in offspring, besides having a local mucosal immune defence effect 540 in mammae of the sow. Oh et al. (2012) demonstrated passive transfer of maternally 541 derived PCV-2-specific cellular immune response to piglets from colostrum, by 542 measuring intradermal delayed type hypersensitivity responses and specific blood 543 lymphocyte proliferation in piglets from vaccinated sows. For the other swine 544 pathogenic mycoplasma, Mycoplasma hyopneumoniae, Bandrick et al. (2008, 2014) 545 have described the transfer of functional antigen-specific T-cells from sows to their 546 offspring that leaves the newborn piglet able to mount an antigen-specific secondary 547 immune response. They further stated that this transfer of Mycoplasma 548 hyopneumoniae-specific cellular immunity is dependent on the piglet suckling its 
549 biological mother sow (Bandrick et al., 2011). Something similar could take place 550 between $M$. hyosynoviae infected sows and their offspring too.

552 We have earlier induced clinical M. hyosynoviae arthritis experimentally in 6-week553 old piglets from a mycoplasma free herd (Lauritsen et al., 2008), and the findings in 554 the NC group confirms the absence of a strictly age related insusceptibility to $M$. 555 hyosynoviae arthritis in young pigs. Regardless of whether it is primarily acquired 556 antibodies (and cells) or also an active immune response that causes the high level of 557 protection in the CCE group, it is evident that the general absence of arthritis in herd 558 piglets can be ascribed mainly to their immunological status with respect to $M$. 559 hyosynoviae. Protective immunity against the infection is apparently achievable in 560 piglets, a fact that is of importance e.g. when considering development of an effective 561 immune prophylaxis.

563 Conflict of interest

564 The authors declare that they have no competing interests.

566 Acknowledgements

567 The project was funded by The Research Centre for the Management of Animal 568 Production and Health (CEPROS), Denmark (project no. CEP-SVS97-7). Hans 
569 Skåning and staff in the animal facility at National Veterinary Institute are thanked 570 for their great help. Also laboratory technicians Ulla Amtoft, Parvin Faghan, Jesper 571 Juehl Hansen and Jan Lauritsen are thanked for assistance with Mycoplasma 572 cultivation, cell culture, antigen production, and serological testing. Associate 573 Professor N. C. Nielsen from the University of Copenhagen is thanked for invaluable 574 input on the experimental planning and design. John Lund and his staff from the 575 experimental herd related to Faculty of Health and Medical Sciences, University of 576 Copenhagen, are thanked for supplying colostrum.

583 Bandrick M., Pieters M., Pijoan C., Baidoo S.K., Molitor T.W., 2011. Effect of cross-fostering on 584 transfer of maternal immunity to Mycoplasma hyopneumoniae to piglets. Vet. Rec. 168, 100.

585 Bandrick M., Ariza-Nieto C., Baidoo S.K., Molitor T.W., 2014. Colostral antibody-mediated and 586 cell-mediated immunity contributes to innate and antigen-specific immunity in piglets. Dev. Comp. 587 Immunol. 43, 114-20. 
589 Davis, C.S., 2002. Statistical Methods for the Analysis of Repeated Measurements. Springer, New 590 York, USA.

591 Diggle, P.J., Heagerty, P., Liang, K.-Y., Zeger, S.L., 2002. Analysis of Longitudinal Data. Oxford 592 University Press, Oxford, UK. 400 pp.

593 Dohoo, I., Martin, W., Stryhn, H., 2009. Veterinary Epidemiologic Research. AVC-Inc, 594 Charlottetown, Canada. 865 pp.

595 Evans, P.A., Newby, T.J., Stokes, C.R., Bourne., F.J., 1982. A study of cells in the mammary 596 secretions of sows. Vet. Immunol. Immunopathol. 3, 515-527.

597 Frenyo, V.L., Pethes, G., Antal, T., Szabo, I., 1981. Changes in colostral and serum IgG content in 598 swine in relation to time. Vet. Res. Commun. 4, 275-282.

599 Friis, N.F., Ahrens, P., Larsen, H., 1991. Mycoplasma hyosynoviae isolation from the upper 600 respiratory tract and tonsils of pigs. Acta Vet. Scand. 32, 425-429.

601 Greenland, S., Senn, S.J., Rothman, K.J., Carlin, J.B., Poole, C., Goodman, S.N., Altman, D.G., 602 2016. Statistical tests, P values, confidence intervals, and power: a guide to misinterpretations. Eur J 603 Epidemiol, 31, 337-50

604 Hagedorn-Olsen, T., Nielsen, N.C., Friis, N.F., Nielsen. J., 1999a. Progression of Mycoplasma 605 hyosynoviae infection in three pig herds. Development of tonsillar carrier state, arthritis and 606 antibodies in serum and synovial fluid in pigs from birth to slaughter. Zentralbl. Veterinarmed. A $607 \quad 46,555-564$. 
608 Hagedorn-Olsen, T., Nielsen, N.C., Friis, N.F., 1999b. Induction of arthritis with Mycoplasma 609 hyosynoviae in pigs: clinical response and re-isolation of the organism from body fluids and organs. 610 Zentralbl. Veterinarmed. A 46, 317-325.

611 Hagedorn-Olsen, T., Basse, A., Jensen, T.K., Nielsen, N.C., 1999c. Gross and histopathological 612 findings in synovial membranes of pigs with experimentally induced Mycoplasma hyosynoviae 613 arthritis. APMIS 107, 201-210.

614 Heegaard, P.M., Stockmarr, A., Piñeiro, M., Carpintero, R., Lampreave, F., Campbell, F.M., 615 Eckersall, P.D., Toussaint, M.J., Gruys, E., Sorensen, N.S., 2011. Optimal combinations of acute 616 phase proteins for detecting infectious disease in pigs. Vet Res. 42:50.

617 Hlavova, K., Stepanova, H., Faldyna, M., 2014. The phenotype and activation status of T and NK 618 cells in porcine colostrum suggest these are central/effector memory cells. Vet J. 202, 477-82.

619 Klobasa, F., Werhahn, E., Butler, J.E., 1981. Regulation of humoral immunity in the piglet by 620 immunoglobulin of maternal origin. Res. Vet. Sci. 31, 195-206.

621 Kobisch, M., Friis., N.F., 1996. Swine mycoplasmoses. Rev. Sci. Tech. 15, 1569-1605.

622 Kokotovic, B., Friis, N.F., Ahrens, P., 2002a. Characterization of Mycoplasma hyosynoviae strains 623 by amplified fragment length polymorphism analysis, pulsed-field gel electrophoresis and 16S 624 ribosomal DNA sequencing. J. Vet. Med. B 49, 245-252.

625 Kokotovic, B., Friis, N.F., Nielsen, E.O., Ahrens. P., 2002b. Genomic diversity among Danish field 626 strains of Mycoplasma hyosynoviae assessed by amplified fragment length polymorphism analysis. 627 Vet. Microbiol. 85, 221-231. 
628 Lauritsen, K.T., Hagedorn-Olsen, T., Friis, N.F., Lind, P., Jungersen, G., 2008. Absence of strictly 629 age-related resistance to Mycoplasma hyosynoviae infection in 6-week-old pigs. Veterinary 630 Microbiology 130, 385-390.

631 Magnusson, U., Rodriguez-Martinez, H., Einarsson, S., 1991. A simple, rapid method for 632 differential cell counts in porcine mammary secretions. Vet. Rec. 129, 485-490.

633 Mosmann, T., 1983. Rapid colorimetric assay for cellular growth and survival: application to 634 proliferation and cytotoxicity assays. J. Immunol. Methods 65, 55-63.

635 Nechvatalova, K., Kudlackova, H., Leva, L., Babickova, K., Faldyna, M., 2011. Transfer of 636 humoral and cell-mediated immunity via colostrum in pigs. Vet. Immunol. Immunopathol. 142, 95637100.

638 Nielsen, E.O., Nielsen, N.C., Friis, N.F., 2001. Mycoplasma hyosynoviae arthritis in grower-finisher 639 pigs. J. Vet. Med. A 48, 475-486.

640 Nielsen, E.O., Lauritsen, K.T., Friis, N.F., Enøe, C., Hagedorn-Olsen, T., Jungersen, G., 2005. Use 641 of a novel serum ELISA method and the tonsil-carrier state for evaluation of Mycoplasma 642 hyosynoviae distributions in pig herds with or without clinical arthritis. Vet. Microbiol. 111, 41-50.

643 Oh, Y., Seo, H.W., Han, K., Park, C., Chae, C., 2012. Protective effect of the maternally derived 644 porcine circovirus type 2 (PCV2)-specific cellular immune response in piglets by dam vaccination 645 against PCV2 challenge. J Gen Virol. 93, 1556-62.

646 Rautiainen, E., Wallgren, P., 2001. Aspects of the transmission of protection against Mycoplasma 647 hyopneumoniae from sow to offspring. J Vet Med B Infect Dis Vet Public Health. 48, 55-65. 
648 Razin, S., Yogev, D., Naot, Y., 1998. Molecular biology and pathogenicity of mycoplasmas. 649 Microbiol. Mol. Biol. Rev. 62, 1094-1156.

650 Riber, U., Jungersen, G., 2007. Cell-mediated immune responses differentiate infections with 651 Brucella suis from Yersinia enterocolitica serotype 0:9 in pigs. Vet. Immunol. Immunopathol. 116, $652 \quad 13-25$.

653 Rooke, J.A., Bland, I.M., 2002. The acquisition of passive immunity in the new-born piglet. 654 Livestock Production Science 78, 13-23.

655 Ross, R.F., 1973. Predisposing factors in Mycoplasma hyosynoviae arthritis in swine. J. Infect. Dis. 656127 (Supplement), S84-86.

657 Ross, R.F., Duncan, J.R., 1970. Mycoplasma hyosynoviae arthritis of swine. J. Am. Vet. Med. 658 Assoc. $157,1515-1518$.

659 Ross, R.F., Karmon, J.A., 1970. Heterogeneity among strains of Mycoplasma granularum and 660 identification of Mycoplasma hyosynoviae, sp. n. J. Bacteriol. 103, 707-713.

661 Ross, R.F., Spear, M.L., 1973. Role of the sow as a reservoir of infection for Mycoplasma 662 hyosynoviae. Am. J. Vet. Res. 34, 373-378.

663 Ross, R., Grebe, H., Kirchhoff, H., 1978. Serological and electrophoretic characteristics of 664 Mycoplasma hyosynoviae. Zentralbl. Veterinarmed. B 25, 444-451.

665 Salmon, H., 2000. Mammary gland immunology and neonate protection in pigs. Homing of 666 lymphocytes into the MG. Adv. Exp. Med. Biol. 480, 279-286.

667 Salmon, H., Berri, M., Gerdts, V., Meurens, F., 2009. Humoral and cellular factors of maternal 668 immunity in swine. Dev Comp Immunol. 33, 384-93. 
669 Schollenberger, A., Degorski, A., Frymus, T., Schollenberger, A., 1986a. Cells of sow mammary 670 secretions. I. Morphology and differential counts during lactation. Zentralbl. Veterinarmed. A 33, $671 \quad 31-38$.

672 Schollenberger, A., Frymus, T., Degorski, A., Schollenberger, A., 1986b. Cells of sow mammary 673 secretions. II. Characterization of lymphocyte populations. Zentralbl. Veterinarmed. A 33, 39-46.

674 Sibila, M., Bernal, R., Torrents, D., Riera, P., Llopart, D., Calsamiglia, M., Segalés, J., 2008. Effect 675 of sow vaccination against Mycoplasma hyopneumoniae on sow and piglet colonization and 676 seroconversion, and pig lung lesions at slaughter. Vet Microbiol. 127, 165-70.

677 Tuboly, S., Bernath, S., Glavits, R., Medveczky, I., 1988. Intestinal absorption of colostral 678 lymphoid cells in newborn piglets. Vet. Immunol. Immunopathol. 20, 75-85.

679 Wagstrom, E.A., Yoon, K.J., Zimmerman, J.J., 2000. Immune components in porcine mammary 680 secretions. Viral Immunol. 13, 383-397.

681 Wallgren, P., Bölske, G., Gustafsson, S., Mattsson, S., Fossum, C., 1998. Humoral immune 682 responses to Mycoplasma hyopneumoniae in sows and offspring following an outbreak of 683 mycoplasmosis. Vet Microbiol. 60, 193-205.

684 Williams, P.P., 1993. Immunomodulating effects of intestinal absorbed maternal colostral 685 leukocytes by neonatal pigs. Can. J. Vet. Res. 57, 1-8.

686 Yang, H., Oura, C.A., Kirkham, P.A., Parkhouse, R.M., 1996. Preparation of monoclonal anti687 porcine CD3 antibodies and preliminary characterization of porcine T lymphocytes. Immunology. $688 \quad 88,577-85$. 